\title{
THE COMPLEX PROTOSTELLAR SOURCE IRAS 04325+2402 ${ }^{1}$
}

\author{
Lee Hartmann ${ }^{2}$, Nuria Calvet ${ }^{2,3}$, Lori Allen ${ }^{2}$, Hua Chen ${ }^{4}$, and Ray Jayawardhana ${ }^{2}$ \\ Harvard-Smithsonian Center for Astrophysics, 60 Garden St., MS 42, Cambridge, MA 02138; \\ hartmann@cfa.harvard.edu
}

\begin{abstract}
We report Hubble Space Telescope near-infrared NICMOS observations of a remarkable low-luminosity Class I (protostellar) source in the Taurus Molecular Cloud. IRAS $04325+2402$ exhibits a complex bipolar scattered light nebula. The central continuum source is resolved and may be multiple, or may be crossed by a small dust lane. Complex arcs seen in scattered light surround the central source; the physical nature of these structures is not clear, but they may reflect perturbations from multiple stellar sources or from time-dependent mass ejection. A second, resolved continuum source is found at a projected distance of approximately 1150 AU from the central region, near the edge of a nebular lobe probably produced by outflow. The images indicate that this second source is another low-luminosity young stellar object, seen nearly edge-on through a dusty disk and envelope system with disk diameter $\sim 60$ AU. We suggest that the scattered light "streaks" associated with this second source are limb-brightened outflow cavities in the dusty envelope, possibly perturbed by interaction with the outflow lobes of the main source. The nature of the companion is uncertain, since it is observed mostly in scattered light, but is most probably a very low mass star or brown dwarf, with a minimum luminosity of $\sim 10^{-2} L \odot$. Our results show that protostellar sources may have multiple centers of infall and non-aligned disks and outflows, even on relatively small scales.
\end{abstract}

Subject headings: circumstellar matter, stars: formation, stars:pre-main sequence

\footnotetext{
${ }^{1}$ Based on observations with the NASA/ESA Hubble Space Telescope, obtained at the Space Telescope Science Institute, which is operated by the Association of Universities for Research in Astronomy, Inc., under NASA contract No. NAS5-26555.

${ }^{2}$ Visiting astronomer at the NASA Infrared Telescope Facility, which is operated by the University of Hawaii under contract to the National Aeronautics and Space Administration.

${ }^{3}$ also Centro de Investigaciones de Astronomía, Mérida, Venezuela

${ }^{4}$ Currently at N.E.T., 6500 Paseo Padre Parkway, Fremont, CA 94555
} 


\section{INTRODUCTION}

Understanding the manner in which protostellar envelopes collapse to stellar and disk systems is an essential problem in star formation. While progress has been made in testing the predictions of the standard theory of protostellar collapse using overall spectral energy distributions (SEDs) and source statistics of protostellar (Class I) sources in the Taurus molecular complex (e.g., Adams, Lada, \& Shu 1987; Butner et al. 1991; Kenyon, Calvet, \& Hartmann 1993 = KCH; Kenyon et al. 1990, 1994), many details remain to be understood. In particular, the roles of outflows and non-spherical initial cloud structure in shaping protostellar clouds (Whitney \& Hartmann 1993;

Kenyon et al. 1993; Calvet et al. 1994; Hartmann et al. 1994, 1996; Li \& Shu 1996, 1997), and the way in which these effects might or might not combine with the angular momentum distribution of protostellar clouds to produce collapse to binary systems and disks (cf. Terebey, Shu, \& Cassen 1984; Boss 1995, and references therein), is far from clear.

Near-infrared imaging observations can play an important role in help resolving these problems. While near-IR images are sensitive to very small amounts of material, due to the efficiency of dust scattering and absorption, such observations can be crucial in indicating the positions and properties of the central stars, and in tracing envelope structure on small scales difficult to observe in other spectral regions.

Here we report Hubble Space Telescope near-infrared imaging observations of a Taurus protostellar (Class I) source, IRAS 04325+2402 (Beichman et al. 1986). This is a low-luminosity source $\left(\log L / L_{\odot}=-0.14\right)$ with other properties typical of Class I Taurus sources $(\mathrm{KCH})$. This source is multiple, and exhibits a complex and confusing morphology, illustrating the limits of SED analysis for understanding protostellar sources.

\section{OBSERVATIONS AND DATA REDUCTION}

Near-infrared observations of IRAS $04325+2402$ were obtained with the HST NICMOS Camera 2 on 11/11/97 UT. Images were obtained with the F110W, F160W, F205W, and F212N filters, the latter to search for $\mathrm{H}_{2}$ emission. A five-position dither pattern with a separation of approximately 6 arcsec was used for the wide filters. Because the source is large and was not well-centered, only four of the 5 dithered images were useful in producing the final images. Dark sky images for F205W were obtained with a single chop position offset by 40 arcsec. Exposure times were $128 \mathrm{sec}$ per frame in F205W and $40 \mathrm{sec}$ in F160W. The $80 \mathrm{sec}$ F212N exposures provided no hint of excess $\mathrm{H}_{2}$ emission, and the 40 sec $\mathrm{F} 110 \mathrm{~W}$ exposures showed only very faint nebulosity, so we do not consider them further.

The data were reduced using the standard "calnica" routine in the STSDAS packages with 
updated calibration files and other tasks within IRAF. Offsets for the construction of the final image were derived by cross-correlating image features. Final images were created in two ways. First, we translated the images using fractional pixel shifts using the IRAF "imshift" task, and constructed a median combined image using the "imcombine" task. Second, images were combined using the "drizzle" task in IRAF/STSDAS (Fruchter \& Hook 1999). "Drizzle" employs a variable-pixel linear reconstruction algorithm, for the re-sampling of undersampled, dithered data, while correcting for geometric distortions and preserving photometry. In this method, pixels are mapped from an input image, or group of input images, into pixels in a subsampled output image. In this application, the input pixels were shrunk to $90 \%$ of their original size (pixfrac $=\mathbf{0 . 9}$ ) before being drizzled onto a grid with pixels half the original pixel size (pixscale $=\mathbf{0 . 5}$ ). Weighted image masks, taking into account bad pixels and the coronographic spot, were prepared for each image and used in the drizzle. The images resulting from these two procedures are extremely similar. For basic image display we show the drizzled versions; for comparison with models ( $₫ 4)$, we use the imcombined images to maintain the original pixel scale of the observations.

Supporting images at J, H, K, and an $\mathrm{H}_{2} 2.12 \mu$ m filter were obtained with NSFCAM on the NASA IRTF telescope on 26 and 27 Nov 1998. Data were obtained on two pixel scales, 0.15 and $0.3 \mathrm{arcsec} / \mathrm{pixel}$, on successive nights. The data were reduced with standard IRAF routines.

\section{RESULTS}

\subsection{Images}

Figure 1 shows the large-scale K-band nebulosity as observed at IRTF with NSFCAM. The large-scale bipolar structure is evident, consistent with typical scattered light structures produced by bipolar outflows in young stellar objects. The northern scattered light lobe is much brighter (and perhaps more conical) than the southern lobe, and exhibits two bright structures toward its apex, in agreement with the K' image presented by Hodapp (1994). An anomalous bright "knot", which we refer to as object C, is apparent near the western edge of the northern lobe (as also shown in the image of Hodapp), and is crossed by a curved bright streak proceeding from SE to NW which apparently passes through the knot. The streaks exhibit a peculiar "corkscrew" appearance; there may be some corresponding structure, though not symmetrically placed, in the southern lobe. A comparison with the narrow $\mathrm{H}_{2}$ filter images shows that there is no measurable excess relative to either the broad-band $\mathrm{K}$ filter or a neighboring continuum filter, consistent with the F $212 \mathrm{~N}$ images. Thus, the spectrum of $\mathrm{C}$ is not dominated by $\mathrm{H}_{2}$ emission, and must be mostly continuum.

The NICMOS F205W composite drizzled image is shown in Figure 2. To preserve resolution

\footnotetext{
${ }^{5}$ Distributed by NOAO, which is operated by AURA, Inc. under cooperative agreement with the National Science Foundation.
} 
the image has not been rotated from the observed position angle of $38^{\circ}$ for the y axis. The bright central region of the previous image is now resolved into a bright, extended central object, which we call $\mathrm{A} / \mathrm{B}$. Immediately $\mathrm{S}$ of $\mathrm{A} / \mathrm{B}$ there is a bright ring or arc; to the $\mathrm{N}$ there is a peculiar $\Sigma$-shaped scattered light nebulosity. Object A/B is not a point source; as shown in the expanded view in Figure 3a, there is a double structure, possibly indicating two sources, or possibly indicating a dark absorption lane running roughly EW across the object.

Figure 3b shows an enlargement of the F205W image near object C. The object is clearly resolved into a double-lobed emission separated by a dark lane. This structure is present in the three individual dithered images in which $\mathrm{C}$ appears. This image structure is reminiscent of the HST WFPC2 images of HH30 (Burrows et al. 1996) and of the faint companion to HK Tau (Stapelfeldt et al. 1998), which have been interpreted as scattered light from the upper surfaces of edge-on, flared disks around T Tauri stars, possibly augmented by a dusty, relatively tenuous, envelope (Wood et al. 1998).

Figure 4 is a false-color rendition of IRAS $04325+2402$ made by combining the F205W (red) and the F160W (green) images. The $\mathrm{W}$ portion of the knot is relatively brighter in F205W than F160W, as indicated by the color scheme, suggesting greater extinction as would be expected in a disk/envelope model $(\S 4)$. In other respects the F160W image closely parallels the F205W image structure, but with substantially lower signal-to-noise.

Table 1 gives positions of components $\mathrm{A} / \mathrm{B}$ and $\mathrm{C}$. The position given for A/B is actually the position of $\mathrm{A}$, the brightest portion ( $\mathrm{S}$ component) of the extended object (Figure 3a); component $\mathrm{B}$ is approximatley 0.074 arcsec east and 0.23 arcsec north of $\mathrm{A}$.

\subsection{Photometry}

Determination of fluxes and conversion to magnitude systems is complicated by the difficulty of subtracting spatially-varying background, by the very red colors of these objects, and by the non-standard nature of the NICMOS filters. For the NICMOS data we used aperture corrections derived from observations of a very red standard star (Oph S-1) kindly provided by Marcia Rieke; these aperture corrections agreed well with results from the Tiny Tim program (Krist \& Hook 1997). The fluxes reported in Table 1 use the standard calibration without any attempt to correct for color. Usually color terms are ignored in determining fluxes and magnitudes, as we do here, but we note that color corrections could be significant for such red objects. The corresponding magnitudes reported in Table 2 adopt the Vega zero point values.

At IRTF, we measured a K magnitude (in the UKIRT system) of approximately $11.23 \pm 0.05$ in a 7.2 arcsec diameter aperture centered on the main source (Table 2). This is consistent with the $\mathrm{K}=11.27$ reported by Kenyon et al. (1993) in an 8 arcsec diameter aperture; however, we measured an $(\mathrm{H}-\mathrm{K})=1.79$ (UKIRT) instead of the $(\mathrm{H}-\mathrm{K})=2.05$ reported by KGWH. Our color results must be viewed with some caution, however, because there is a large color 
term in the transformation of $(\mathrm{H}-\mathrm{K})$ between the IRTF system and the UKIRT or CIT systems $((\mathrm{H}-\mathrm{K})[\mathrm{IRTF}] \sim 0.8(\mathrm{H}-\mathrm{K})[\mathrm{UKIRT}, \mathrm{CIT}])$. This color transformation has been derived for stars which are much bluer than the red systems studied here, and so may not be appropriate to apply to IRAS $04325+2402$.

The brightness of object $\mathrm{C}$ is uncertain for reasons of the difficulty in background subtraction. The F205W magnitude reported in Table 2 is considerably fainter than the $\mathrm{K}$ magnitude from IRTF, but the difference can probably be understood due to the difference in central wavelengths of the two filters, and the difficulty of subtracting out the nebulous background at low spatial resolution. (We used a larger aperture for the IRTF measurement because of seeing limitations.)

\section{DISCUSSION}

\subsection{Object $\mathrm{C}$ - nebula}

The image of Object $\mathrm{C}$ exhibits similarities to other HST images of young stellar objects surrounded by circumstellar material in the form of disks and/or envelopes, such as HH30 (e.g., Burrows et al. 1996). To investigate this possibility further, we constructed a series of models of disks illuminated by the central star, with and without infalling envelopes. Given the limited observational information available (for example, the luminosity and mass of the central star are unknown), and the number of parameters needed for a disk plus envelope system, no unique model can be constructed. Instead we adopt properties and parameters typical of models of Class I sources and T Tauri disks to investigate the plausibility of our overall interpretation.

The envelope is taken to have the density structure of the standard protostellar infall model of Terebey, Shu, and Cassen (1984). The radiative equilibrium temperature of the envelope is calculated as in $\mathrm{KCH}$ and Calvet etal. (1994). The parameters that describe the envelope are $\rho_{1}$, the density the envelope would have at $1 \mathrm{AU}$ if it were purely infalling radially (i.e., if the material had no angular momentum), and the centrifugal radius $R_{c}$. The density parameter is proportional to the mass infall rate $\dot{M}, \rho_{1}=5.3 \times 10^{-14} \mathrm{~g} \mathrm{~cm}^{-3}\left(\dot{M} / 10^{-5} M_{\odot} y r^{-1}\right)\left(M_{*} / M_{\odot}\right)^{-1 / 2}$. The disk is assumed to be physically thin and vertically isothermal, with a radial temperature distribution $T=10 K(100 \mathrm{AU} / R)^{1 / 2}$, where $\mathrm{R}$ is the cylindrical radius, and surface density distribution $\Sigma=\Sigma_{0}\left(R_{*} / R\right)$, where $R_{*}$ is the stellar radius, as suggested by the detailed models of D'Alessio et al. $(1998,1999)$. The disk has a fixed outer radius given by $R_{c}$, and its density distribution along the $\mathrm{z}$ axis at fixed radius is determined from the equation of hydrostatic equilibrium.

To calculate an image of the system for a given inclination to the line of sight, we solve the transfer equation for the specific intensity along rays that cross the system at fixed projected coordinates on the plane of the sky. The emissivity at each point along the ray is given by the sum of two terms (1) direct stellar light, attenuated by the intervening material between the star and the point and scattered towards the observer with a Henyey and Greenstein phase function 
$(g=0.03)$ (cf. Calvet et al. 1992), and (2) isotropic scattering towards the observer of the diffuse radiation field in the envelope. This diffuse field is obtained directly from the solution of the radial radiative transfer equations in the envelope (cf. Calvet et al. 1994). The first term corresponds to the single scattering approximation, which describes well the behavior of the photons scattered by the disk (Burrows et al. 1996). The second term represents multiple scattering in the envelope, in the spherically symmetric approximation. This approximation becomes more accurate at large distances, where the envelope becomes spherical. Draine and Lee (1984) dust properties have been assumed. The theoretical specific intensity map was binned into pixels of 0.038 " x 0.038 ", i.e., half the original image pixel scale and convolved with the HST PSF function derived from Tiny Tim (Krist \& Hook 1997). After convolution, the images were rebinned back to the original pixel scale to compare with observations.

Our best envelope + disk model for Object $\mathrm{C}$ is shown in Figure 5 compared with the F205W image. The disk has a radius of $30 \mathrm{AU}$, and it is immersed in a low density envelope characterized by $\rho_{1}=10^{-14} \mathrm{~g} \mathrm{~cm}^{-3}$ and $R_{c}=30 \mathrm{AU}$. The system is viewed at an inclination angle to the line of sight of $83^{\circ}$. The theoretical image contours agree reasonably well with the observed image contours, though we cannot reproduce the observed non-axisymmetric features with an axisymmetric model.

Our investigation indicates that neither pure disk models nor pure envelope models of the type considered here can satisfactorily reproduce the observed image. Models which include disks alone (left panel in Figure 6) do not have emission extending sufficiently far in the perpendicular direction to the disk plane (i.e., the disk image is too "flat"). Conversely, pure TSC infall models do not exhibit sufficient indication of a dark lane (middle panel of Figure 6). The situation is reminiscent of that of models for HH30 by Wood et al. (1998), in which the brightest structures were produced by a disk but the faint, highly-extended scattered light structures were attributed to an envelope.

The model calculations for F160W are in approximate agreement with the observations. We do not attempt a detailed comparison because of the substantial noise in this image. The model extinction is (slightly) higher toward the southeastern component, in qualitative agreement with the (modest) color difference observed. Assuming an intrinsic color of the central object of $(\mathrm{H}-\mathrm{K})=0.6 \pm 0.3($ see $\S 4.2)$, the model predicts $(\mathrm{H}-\mathrm{K}) \sim 1.8 \pm 0.3$. Given the uncertainties in the data and the unknown nature of the colors of the central source, this agreement is sufficiently close to demonstrate the general plausibility of the model.

We conclude that a model which includes both a disk and an envelope can qualitatively account for the image of this object. This model also may explain the scattered light "streaks" or structures associated with C. We suggest that these streaks might delineate the edges of an outflow cavity produced by C (see Figure 7).

If we assume a stellar mass of $0.1 M_{\odot}$ for $\mathrm{C}(\S 4.2)$, the implied infall rate for the envelope of our model is $\sim 6 \times 10^{-7} M_{\odot} y r^{-1}$. This is below the average infall rate of $\sim 4 \times 10^{-6} M_{\odot} y r^{-1}$ estimated 
for typical Taurus Class I sources, and lower than the overall $\sim 1.3 \times 10^{-5} M_{\odot} y r^{-1}\left(M / 0.5 M_{\odot}\right)^{1 / 2}$ derived for the main source in IRAS $04325+2402$ by $\mathrm{KCH}$ (though substantially larger than the $3 \times 10^{-8} M_{\odot} y r^{-1}$ adopted by Wood et al. [1998] for their HH30 model). From the observed source morphology it appears plausible that $\mathrm{C}$ is encountering a lower-density environment than typical of the regions near $\mathrm{A} / \mathrm{B}$, possibly as a result of orbital motion away from the main central mass.

\subsection{Object $\mathrm{C}$ - central star}

The nature of the central star in $\mathrm{C}$ is difficult to determine given the complications involved, but we can make some estimates. For this purpose we use the ground-based IRTF measurements because they are (approximately) on a standard magnitude system, though the lower spatial resolution makes extraction of the source with respect to the nebular background more difficult. The very red $(\mathrm{H}-\mathrm{K})=1.95$ color of $\mathrm{C}$ indicates that the central star must be heavily reddened. The intrinsic colors of (M-type) Classical $\mathrm{T}$ Tauri stars in Taurus (young stars with inner circumstellar disks) range from about $(\mathrm{H}-\mathrm{K}) \sim 0.3$ to $\sim 0.9$ (Meyer et al. 1997) (for objects with no contribution from a dusty envelope). Adopting an intrinsic color $(\mathrm{H}-\mathrm{K})=0.6 \pm 0.3$, the excess is $\mathrm{E}(\mathrm{H}-\mathrm{K})=1.3 \pm 0.3$. Using $\mathrm{A}(\mathrm{K})=1.4 \mathrm{E}(\mathrm{H}-\mathrm{K})$ from Cohen et al. (1981), and ignoring the difference in colors between CIT and UKIRT systems, we find $\mathrm{A}(\mathrm{K})=1.8 \pm 0.4$, so $\mathrm{K}_{\circ}=12.8 \pm 0.4$. Using the results of Wilking, Greene, \& Meyer (1999; their Figure 1) for evolutionary tracks, and adopting an age of $3 \times 10^{5} \mathrm{yr}$ as typical of Class I sources (Kenyon et al. 1990, 1994), the result is a luminosity of $\sim 10^{-2} L_{\odot}$ and a mass of $\sim 0.03-0.02 M_{\odot}$, well into the brown dwarf range.

However, this estimate is almost certainly a lower limit to the stellar luminosity (and therefore to the stellar mass) because the object is undoubtedly seen entirely in scattered light, and thus the observed colors underestimate the extinction directly toward the central star. For the model shown in Figure 6, the observed flux at $\mathrm{K}$ is approximately $4 \%$ of the true stellar flux. Using this factor to correct the observed $\mathrm{K}$ magnitude would result in $K_{\circ} \sim 11$ and a mass of about $0.06 M_{\odot}$ from the Wilking et al. (1999) estimates. Small changes in envelope parameters easily could increase the true $\mathrm{K}$ brightness by substantial factors. At the other extreme, if we assume that $\mathrm{C}$ supplies all of the heating responsible for the IRAS long-wavelength emission (cf. $\mathrm{KCH}$ ), then the luminosity indicates a central star of $\sim 0.25 M_{\odot}$ from the evolutionary tracks of D'Antona \& Mazzitelli (1997). Given that the main source A/B seems to be responsible for most of the scattered light in the main nebular lobe, this seems unlikely. Thus the central star in $\mathrm{C}$ is probably either a very low mass star or a brown dwarf; the F205W observations may provide the first image of a protoplanetary disk around a brown dwarf. Infrared spectra should be obtained to better constrain the nature of $\mathrm{C}$. 


\subsection{Object $\mathrm{A} / \mathrm{B}$}

A/B lies near the apex of the main bipolar reflection nebula (Figure 1), and is probably the main luminosity source in the system. This geometrical relation indicates that A/B is probably the source of bipolar ejection which produces the large-scale reflection nebula structure. The alignment of these cavities are very different than the assumed alignment of object $\mathrm{C}$ (see Figure 7). Thus, it appears that even relatively close, and probably coeval, multiple protostellar systems may have significantly different outflow/disk orientations.

The large extinction toward A/B (as indicated by the colors, and by the extreme faintness of the source at $\mathrm{F} 110 \mathrm{~W}$ and $\mathrm{J}$ ), indicates that A/B is also a center of infall for the extended dusty envelope, as envisaged by the models of $\mathrm{KCH}$ and Kenyon et al. (1993b). Since A/B is probably more luminous and thus possibly more massive than $\mathrm{C}$, it would not be surprising if most of the infall is directed toward it rather than toward $\mathrm{C}$. The presence of multiple gravitating centers in this system may account for some of the peculiar nebular structure.

Whether A/B is itself a multiple stellar system, or simply a complex scattered light structure around a single star, is unclear. While we do not offer a model for the peculiar scattered light structures in the vicinity of this source, we note that they appear to lie along portions of the outflow cones of the main source. The scattered light images may indicate complicated interactions of a structured infall region with outflow (Figure 7), although the precise way this might happen is far from clear.

The lower (southern) lower scattered-light arc is reminiscent of ground-based images of circumbinary dust rings around T Tauri stars in Taurus, as for example is observed in GG Tau (Roddier et al. 1994; see also Dutrey, Guilloteau, \& Simon 1994) and UY Aur (Close et al. 1998). The inner ring radii in these two objects are around $200 \mathrm{AU}$ and $500 \mathrm{AU}$, respectively; for object A, the diameter of the southern ring is about $1.3 \operatorname{arcsec} \sim 180 \mathrm{AU}$ at $140 \mathrm{pc}$. In this picture, the ring could be produced by the tidal action of an orbiting binary or multiple protostellar system. A possible problem with this picture is that A/B does not seem to lie inside of the ring, but somewhat above it.

Object A/B is probably the main gravitating center toward which most of the dusty envelope is presumably collapsing. The model of $\mathrm{KCH}$, which was adjusted to fit the sparsely-observed

long-wavelength SED of the object, suggested an infall rate of $\sim 1.3 \times 10^{-5} M_{\odot} y r^{-1}\left(M / 0.5 M_{\odot}\right)^{1 / 2}$. Even with a large centrifugal radius, $R_{c}=300 \mathrm{AU}$, to reduce extinction toward the main source, the model did not account for the observed near-infrared emission. The attempt of Kenyon et al. (1993) to explain the near-infrared fluxes and large-scale image including outflow cavities in the collapse model led them to estimate $\dot{M} \sim 4-6 \times 10^{-6} M_{\odot} y r^{-1}\left(M / 0.5 M_{\odot}\right)^{1 / 2}$ and $R_{c} \sim 50-200 \mathrm{AU}$. Given the complex scattered light structure seen in our images on scales of $100 \mathrm{AU}$ and less, it is clear that any estimate of the centrifugal radii of collapsing envelopes from near-infrared fluxes alone is very uncertain. 


\subsection{Overall structure}

The peculiar "corkscrew" structure in the northern lobe of the main nebula may be the result of interacting flows of $\mathrm{C}$ and $\mathrm{A}$. In this connection we note that the observed (monopolar) $\mathrm{CO}$ outflow (Heyer et al 1987) in the region is oriented to the northwest of IRAS $04325+2402$. With a position angle of about $-45^{\circ}$, the outflow is closer to the orientation expected for an outflow from $\mathrm{C}$, and completely inconsistent with the nearly north-south orientation of the main scattered light nebula (Figure 1). However, the $\mathrm{CO}$ outflow extends over such a large scale that its connection with IRAS 04325+2402 is unclear; moreover, the observed CO motions are redshifted, whereas one might expect to observe a blueshifted lobe from object $\mathrm{C}$ (cf. Figure 7).

In many respects IRAS $04325+2402$ resembles another Class I Taurus protostar with similar bolometric luminosity and near-IR colors, IRAS 04361+2547 (KCH), which has recently been imaged with NICMOS by Terebey et al. (1998). IRAS 04361+2547 also shows a complex central structure, scattered light "streaks", and a faint companion. Terebey et al. interpret their complex central structure to mean that the main source is a binary. As mentioned above, this may apply to object A as well, though we are hesitant to make a firm interpretation since the possibility also exists that these are simply scattered light structures intersected by absorbing dust lanes.

The point-source companion found by Terebey et al. in $04361+2547$ also lies in a scattered light structure, at a similar distance from the main region (1400 AU vs. the $1150 \mathrm{AU}$ projected distance between objects $\mathrm{A} / \mathrm{B}$ and $\mathrm{C}$ ). This companion has roughly similar colors to object $\mathrm{C}$ but is about a factor of 10 fainter. Terebey et al. estimated the companion mass as $2-5 M_{J}$, and suggested that its position at the end of a scattered light filament might be caused by ejection from the central region over the last $1000 \mathrm{yr}$. Our object $\mathrm{C}$ differs in that it is clearly too bright

to be a $M \lesssim 10 M_{J}$ planet and has its own solar-system sized disk. Another difference is that the streaks or filaments do not point back to A/B, unlike the situation in $04361+2547$. If object C's disk had been a factor of two or so smaller, it would have appeared to be a point source, raising the possibility that the Terebey et al. object might also appear anomalously faint for its color as a result of disk/envelope extinction.

\section{Conclusions}

The Class I Taurus source IRAS $04325+2402$ has been shown to be a multiple system, with at least one additional protostar lying a projected distance of $\sim 1150$ AU from the central source, and that central source may itself be multiple. The NICMOS images of the companion are consistent with the typical model of a low-mass protostar, in which infalling material from a rotating envelope lands upon, and builds up, a disk. The dust envelope structure around the main source appears more complex, but suggests an independent center of collapse. The orientation of the main bipolar cavities seen in scattered light, probably driven by outflow from the main source, lies at a substantial angle to the presumed disk plane of the companion object. This suggests that 
the orientation of disk systems can be quite different even in relatively close multiple protostellar systems.

We wish to thank Bill Vacca for his very helpful support at IRTF, Karla Peterson and Louis Bergeron at STScI, and Marcia Rieke for providing standards. This work has been supported in part by NASA through grant number GO-07413.01-96A from the Space Telescope Science Institute. 
TABLE 1

NICMOS POSITIONS AND PHOTOMETRY

\begin{tabular}{lccccr}
\hline Object & $\alpha(\mathrm{J} 2000)$ & $\delta(\mathrm{J} 2000)$ & F110W $(\mu \mathrm{Jy})$ & F160W $(\mathrm{mJy})$ & F205W $(\mathrm{mJy})$ \\
\hline $\mathrm{A} / \mathrm{B}^{\mathrm{a}}$ & 43535.37 & 240819.5 & $93 \pm 1$ & $3.1 \pm 0.3$ & $14.4 \pm 0.5$ \\
$\mathrm{C}$ & 43535.29 & 240827.6 & $<20$ & $0.14 \pm 0.02$ & $0.47 \pm 0.05$ \\
\hline
\end{tabular}

${ }^{a}$ Position given for A. B is $0.07 \operatorname{arcsec} \mathrm{E}, 0.23 \operatorname{arcsec} \mathrm{N}$ of $\mathrm{A}$.

TABLE 2

IRTF and NICMOS MAGNITUDES

\begin{tabular}{lccccc}
\hline Object & $\mathrm{K}$ & $\mathrm{H}-\mathrm{K}$ & $\mathrm{m}(\mathrm{F} 110 \mathrm{~W})^{\mathrm{a}}$ & $\mathrm{m}(\mathrm{F} 160 \mathrm{~W})^{\mathrm{a}}$ & $\mathrm{m}(\mathrm{F} 205 \mathrm{~W})^{\mathrm{a}}$ \\
\hline $\mathrm{A} / \mathrm{B}^{\mathrm{b}}$ & $11.23 \pm 0.05$ & $1.79 \pm 0.05$ & $18.3 \pm 0.2$ & $13.89 \pm 0.1$ & $11.80 \pm 0.05$ \\
$\mathrm{C}^{\mathrm{c}}$ & $14.61 \pm 0.1$ & $1.95 \pm 0.1$ & $>20$ & $17.27 \pm 0.2$ & $15.52 \pm 0.15$ \\
\hline
\end{tabular}

${ }^{a}$ Magnitudes on HST Vega system.

${ }^{b}$ Magnitudes in 7.2 arcsec diameter apertures.

${ }^{c}$ Magnitudes in 1.37 arcsec diameter aperture (NICMOS) and 1.8 arcsec diameter aperture (IRTF). 


\section{REFERENCES}

Adams, F.C., Lada, C.J., \& Shu, F.H. 1987, ApJ, 321, 788

Beichman, C.A., Myers, P.C., Emerson, J.P., Harris, S., Mathieu, R., Benson, P.J., \& Jennings, R.E. 1986, ApJ, 307, 337

Boss, A.P. 1995, in Circumstellar Disks, Outflows, and Star Formation, RevMexAA (Serie de Conferencias) 1, 165

Burrows, C.J., Stapelfeldt, K.R., Watson, A.M., Krist, J.E., Ballester G.E., Clarke, J.T. Crisp, D., Gallagher III, J.S., Griffiths, R.E., Hester, J.J., Hoessel, J.G., Holtzman, J.A., Mould, J.R., Scowen, P.A., Trauger, J.T., \& Westphal, J.A. 1996, ApJ, 473, 437

Butner, H.M., Evans, N.J. II, Lester, D.F., Levreault, R.M., \& Strom, S.E. 1991, ApJ, 376, 636

Calvet, N., Canto, J., Binette, L., \& Raga, A. 1992, RMxAA, 24, 81

Calvet, N., Hartmann, L., Kenyon, S.J., \& Whitney, B.A. 1994, ApJ, 434, 330

Close, L.M., Dutrey, A., Roddier, F., Guilloteau, S., Roddier, C., Duvert, G., Northcott, M., Ménard, F., Graves, J.E., \& Potter, D. 1998, ApJ, 499, 883

Cohen, J.G., Frogel, J.A., Persson, S.E., \& Elias, J.H. 1981, ApJ, 249, 481

D’Alessio, P., Canto, J., Calvet, N., \& Lizano, S. 1998, ApJ, 500, 411

D’Alessio, P., Calvet, N., Hartmann, L., Lizano, S., \& Canto, J.1999, ApJ, (submitted)

D’Antona, F., \& Mazzitelli, I. 1997, Mem. Soc. Astron. Italiana, 68, 4

Draine, B. T., \& Lee, H. M. 1984, ApJ, 285,89

Dutrey, A., Guilloteau, S., \& Simon, M. 1994, A\&A, 286, 149

Fruchter, A.S. \& Hook, R.N. 1999, PASP, submitted

Hartmann, L., Boss, A., Calvet, N., \& Whitney, B. 1994, ApJ, 430, L49

Hartmann, L., Calvet, N., \& Boss, A. 1996, ApJ, 464, 387

Heyer, M.H., Snell, R.L., Goldsmith, P.F., \& Myers, P.C. 1987, ApJ, 321, 370

Hodapp, K-W. 1994, ApJS, 94, 615

Kenyon, S.J., Hartmann, L., Strom, K. M. \& Strom, S. E. 1990, ApJ, 99, 869

Kenyon, S.J., Calvet, N., \& Hartmann, L. 1993, ApJ, 414, 676 (KCH)

Kenyon, S.J., Whitney, B., A., Gomez, M., \& Hartmann, L. 1993, ApJ, 414, 773

Kenyon, S.J., Marzke, R. O. \& Hartmann, L. 1994, ApJ, 108, 251

Krist, J.E., \& Hook, R.N. 1997, The Tiny Tim User's Guide Version 4.4 (Baltimore: STScI)

Li, Z.-Y., \& Shu, F.H. 1996, ApJ, 472, 211

Li, Z.-Y., \& Shu, F.H. 1997, ApJ, 475, 251 
Meyer, M.R., Calvet, N., \& Hillenbrand, L.A. 1997, AJ, 114, 288

Roddier, C., Roddier, F., Northcott, M.J., Graves, J.E., \& Jim, K. 1996, ApJ, 463, 326

Stapelfeldt, K.R., Krist, J.E., Ménard, F., Bouvier, J., Padgett, D.L., \& Burrows, C.J. 1998, ApJ, 502, L65

Terebey, S., Shu, F.H., \& Cassen, P. 1984, ApJ, 286, 529

Terebey, S., Van Buren, D., Padgett, D.L., Hancock, T., \& Brundage, M. 1998, ApJ, 507, L71

Whitney, B. \& Hartmann, L. 1993, ApJ, 402, 605

Wilking, B.A., Greene, T.P., \& Meyer, M.R. 1999, AJ, 117, 469

Wood, K., Kenyon, S.J., Whitney, B., \& Turnbull, M. 1998, ApJ, 497, 404 
Fig. 1. - K-band image of IRAS $04325+2402$, obtained at IRTF. North is at the top and the displayed region is 76 arcsec on a side.

Fig. 2.- HST NICMOS F205W image mosaic of 04325+2402. This "drizzled" Camera 2 image (see text) is 15.6 arcsec on a side. The orientation of this and the following HST images is such that the vertical axis lies at a position angle 38 degrees east of north.

Fig. 3.- (a) Expanded view of the central region in Figure 2, 5.1 arcsec (horizontal) by 4.2 arcsec (vertical). (b) Expanded view of the companion from Figure 2, 4.56 arcsec by 3.42 arcsec. The companion is clearly resolved, with a dark lane running roughly vertically across the image (see text). Note that the pixels in these drizzled images are one-half the original pixel scale.

Fig. 4. - False color image of 04325+2402 composed of the F205W (red) and F160W (green) images. One observes that the eastern portion of object $\mathrm{C}$ is redder than the western lobe, in agreement with our model which predicts that the fainter western lobe is extincted by a dusty envelope (see text).

Fig. 5.- Image and contour plot comparison of disk (left), envelope (center), and combined disk and envelope (right) models for the F205W image of Object C. Models have been computed at the same pixel scale as the original, non-drizzled image (see text). Contour levels are at in linear steps of $20 \%$ of the peak.

Fig. 6. - Contour plot comparison of F205W image (upper) with model disk and envelope image from Figure 6 (right)(see text). Here the comparison is made with the median-combined dithered image at the original pixel scale of the observations. Contour units are in linear steps of $10 \%$ of the peak.

Fig. 7.- Schematic diagram of suggested source geometry (see text). The regions exterior to the cavities are filled with dusty gas, some of which is presumed to be falling in toward the two mass centers $\mathrm{A} / \mathrm{B}$ and $\mathrm{C}$. 


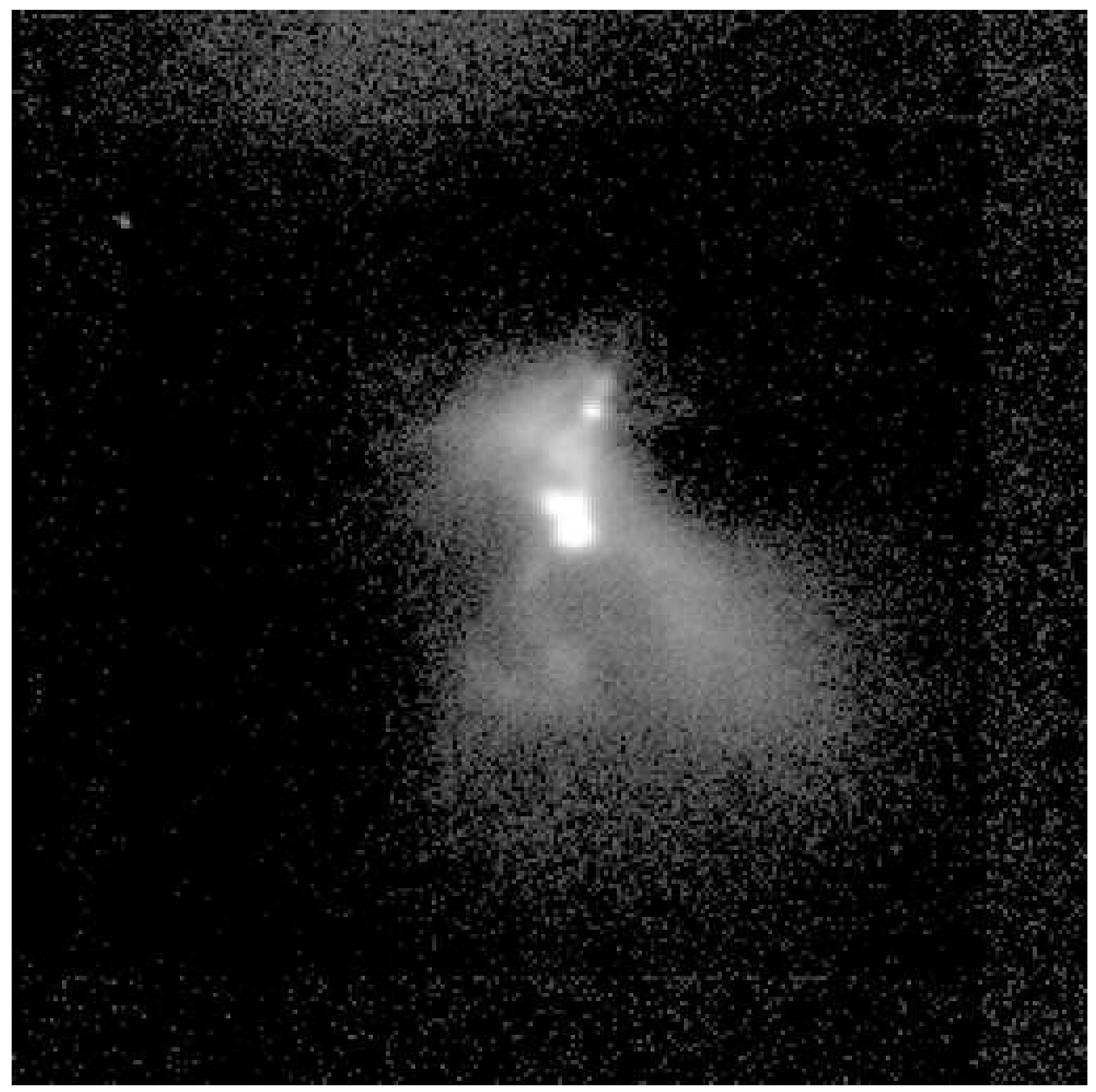




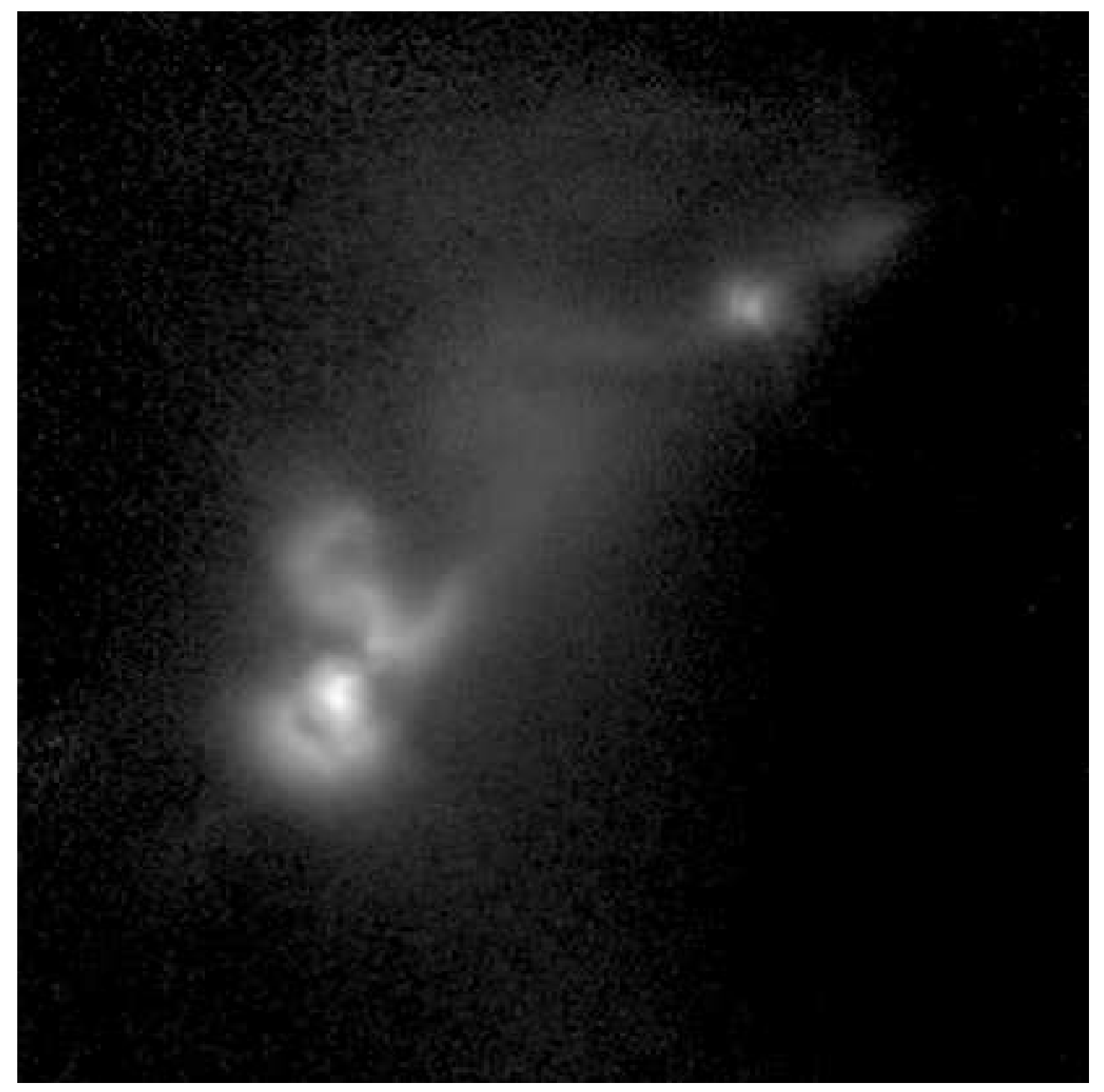




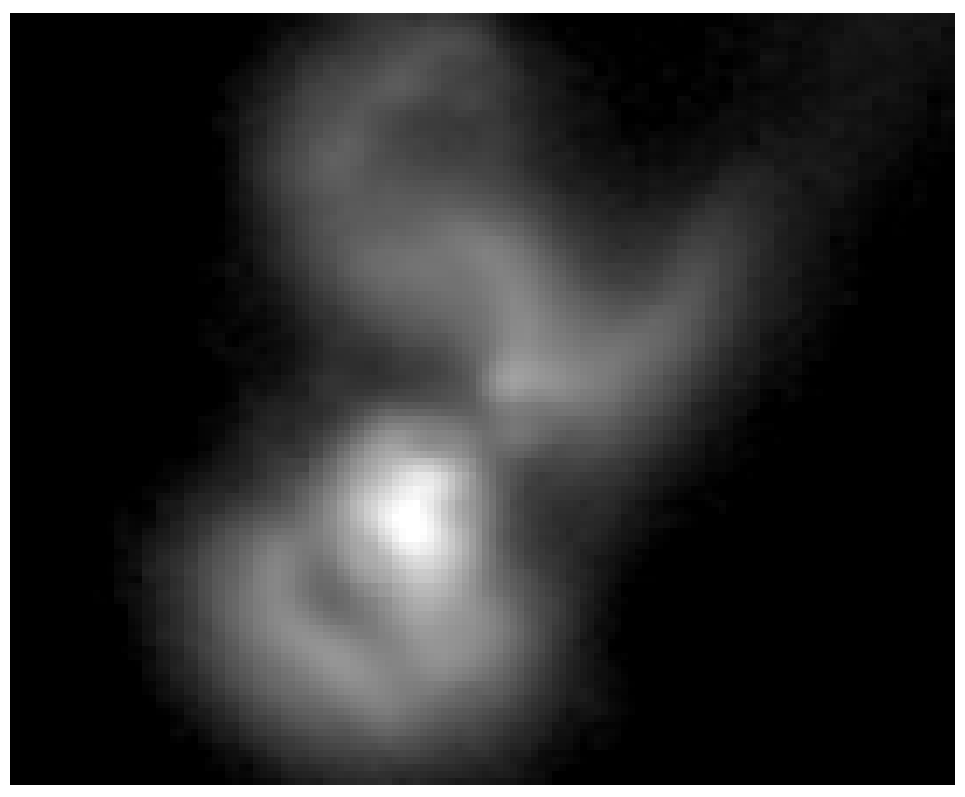




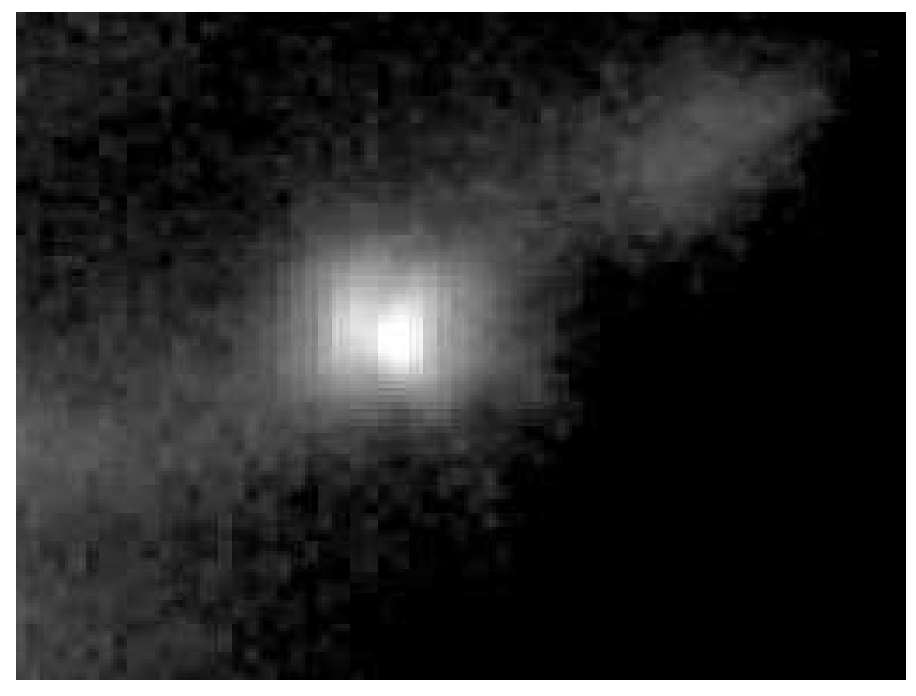




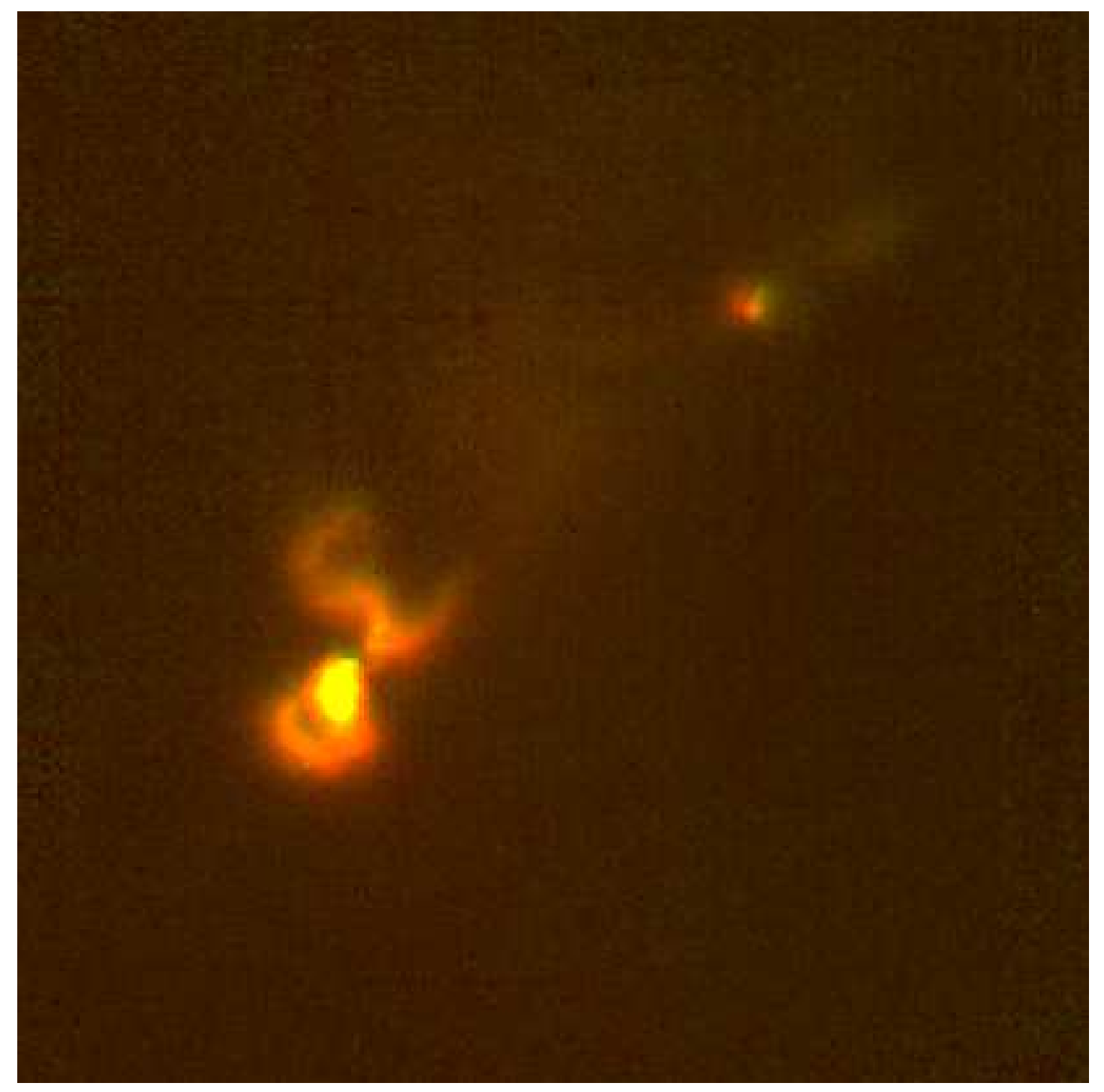




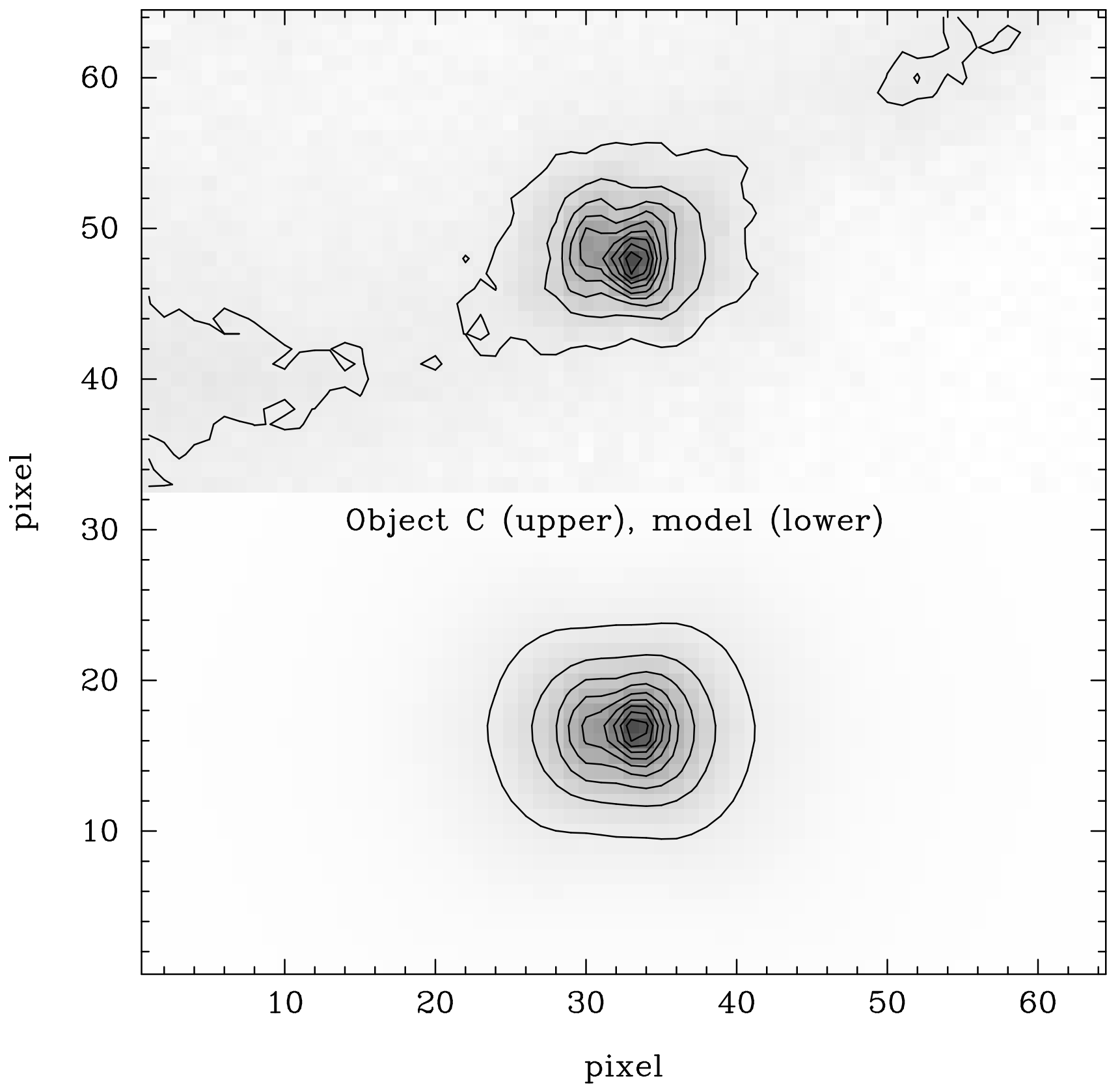




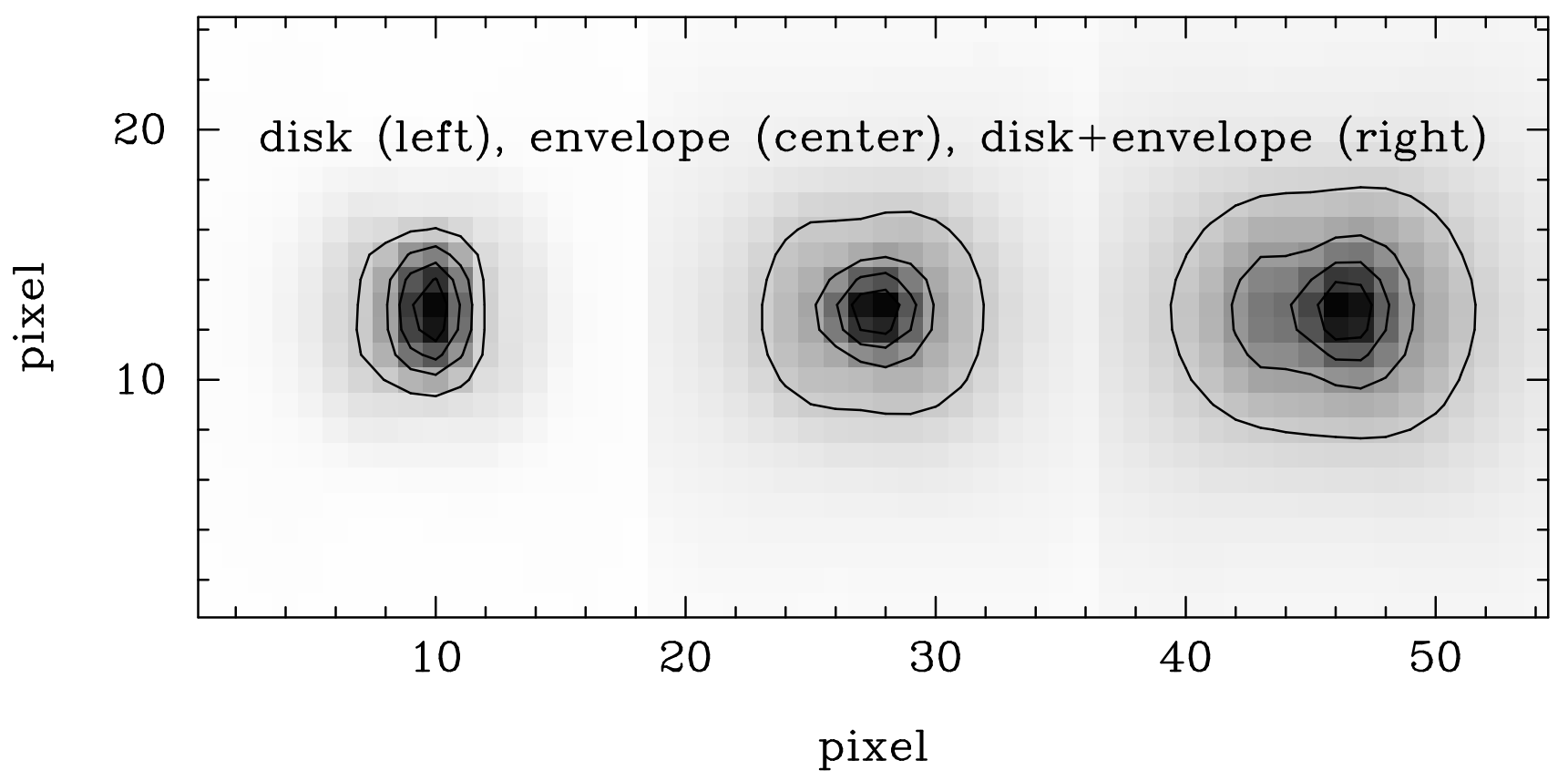



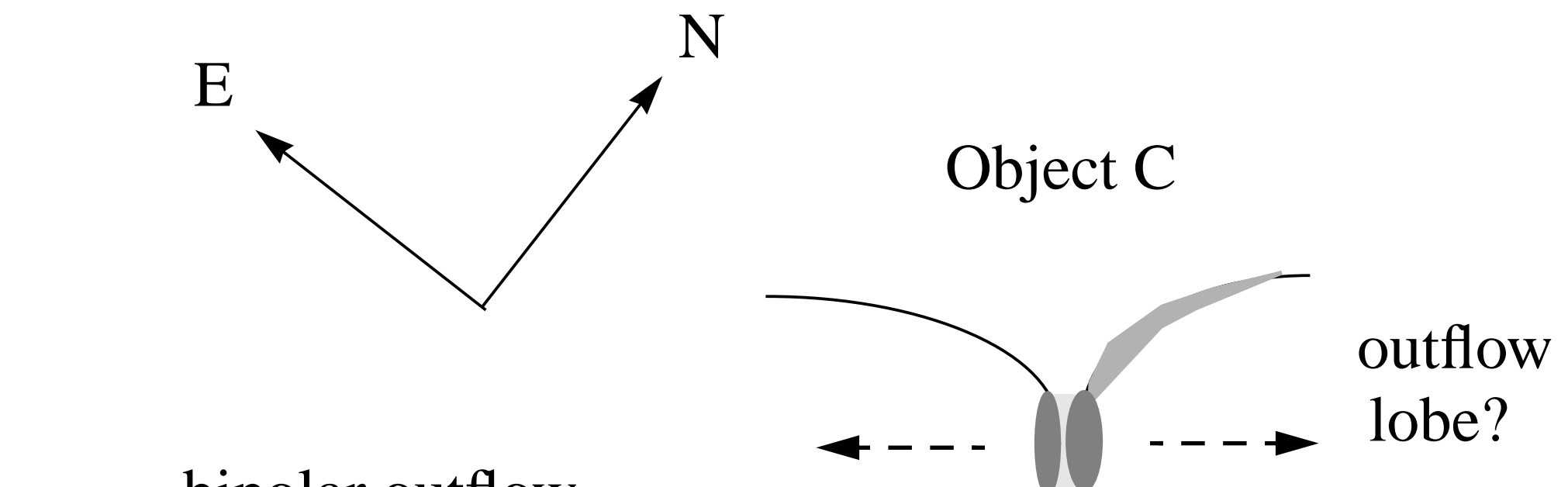

bipolar outflow

cavity (near side)

(from A/B)

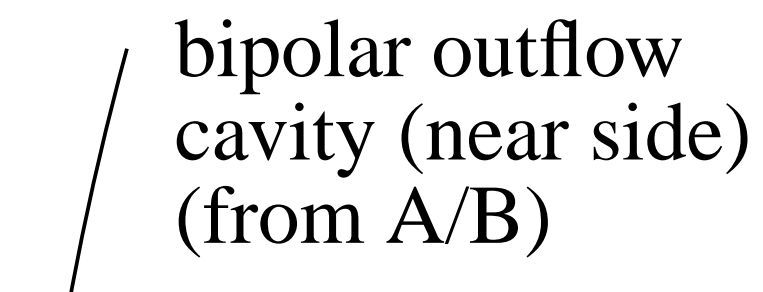

